\title{
The quality of denominator data in surgical site infection surveillance versus administrative data in Norway 2005-2010
}

\author{
Hege Line Løwer ${ }^{*}$, Hanne-Merete Eriksen', Preben Aavitsland ${ }^{2}$ and Finn Egil Skjeldestad ${ }^{3}$
}

\begin{abstract}
Background: High quality of surveillance systems for surgical site infections (SSIs) is the key to their usefulness. The Norwegian Surveillance System for Antibiotic Consumption and Healthcare-Associated Infections (NOIS) was introduced by regulation in 2005, and is based largely on automated extraction of data from underlying systems in the hospitals.

Methods: This study investigates the quality of NOIS-SSI's denominator data by evaluating completeness, representativeness and accuracy compared with de-identified administrative data for 2005-2010. Comparisons were made by region, hospital type and size, age and sex for 4 surgical procedures.

Results: The completeness of NOIS improved from $29.2 \%$ in 2005 to $79.8 \%$ in 2010. NOIS-SSI became representative over time for most procedures by hospital size and type, but not by region. It was representative by age and sex for all years and procedures. Accuracy was good for all years and procedures by all explanatory variables.

Conclusions: A flexible and incremental implementation strategy has encouraged the development of computer-based surveillance systems in the hospitals which gives good accuracy, but the same strategy has adversely affected the completeness and representativeness of the denominator data. For the purpose of evaluating risk factors and implementing prevention and precautionary measures in the individual hospitals, representativeness seems sufficient, but for benchmarking and/or public reporting it is not good enough.
\end{abstract}

Keywords: Electronic surveillance, Register data, Incidence, Infection control, Completeness, Representativeness

\section{Background}

Surveillance of surgical site infections (SSIs) is increasingly regarded as a cornerstone in infection prevention. Many hospitals and countries have successfully implemented surveillance systems [1]. High quality of the systems is a prerequisite for their usefulness. National surveillance of SSIs in Norway was established with the Norwegian Surveillance System for Antibiotic Consumption and Healthcare-Associated Infections (NOIS) Act [2] in 2005, and we have earlier reported in detail on the rationale and functioning of this system [3, 4]. NOIS is based on the Hospitals in Europe Link for Infection Control through Surveillance [5] which was transferred to the European Centre for Disease Prevention and Control (ECDC) [6],

\footnotetext{
* Correspondence: hege.line.lower@fhi.no

${ }^{1}$ Norwegian Institute of Public Health, Department of Infectious Disease Epidemiology, Oslo, Norway

Full list of author information is available at the end of the article
}

and the definitions from the Centers for Disease Control and Prevention's National Healthcare Safety Network [7].

Describing and evaluating the performance of a surveillance system is key to understanding its potential usefulness for public health authorities, hospitals, surgeons and hospital epidemiologists [8]. Validating the quality of the denominator data is important in order to ensure correct incidence rates and proportions. The objective of this study is to investigate denominator data quality by comparing surgical site infection surveillance data from NOIS-SSI with administrative data from the Norwegian Patient Register (NPR). We compare deidentified denominator data for the years 2005-2010 on an aggregated level in order to identify possible discrepancies in terms of completeness, representativeness and accuracy, and to recommend improvements.

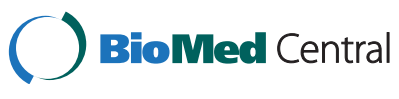

(c) 2015 Løwer et al. Open Access This article is distributed under the terms of the Creative Commons Attribution 4.0 International License (http://creativecommons.org/licenses/by/4.0/), which permits unrestricted use, distribution, and reproduction in any medium, provided you give appropriate credit to the original author(s) and the source, provide a link to the Creative Commons license, and indicate if changes were made. The Creative Commons Public Domain Dedication waiver (http://creativecommons.org/publicdomain/zero/1.0/) applies to the data made available in this article, unless otherwise stated. 


\section{Methods}

NPR was established in 1997 and contains information on all patients who receive specialist health care in Norway. Upon treatment in a hospital, an outpatient clinic or by a contracted private specialist, a series of data are recorded at the treatment site and transmitted to NPR three times a year. The objective of NPR is to form a basis for administration, management and quality assurance in specialist health care services, including financing and funding hospitals [9]. It is considered to be the complete database for hospital care in Norway [10]. NPR-data are harvested electronically from the hospital electronic health records (EHR). It is operated by the Norwegian Directorate of Health. The NPR-data relevant to the present study include variables for all admissions related to the procedure under observation: Patient identifier (de-identified), procedure code (Nordic Medico-Statistical Committee's Classification of Surgical Procedures (NCSP)) [11], dates and times of admission, discharge and procedure, year of birth, sex, and hospital identifier.

NOIS was established in 2005 and is a national, mandatory surveillance system for health-care institutions [2]. The objective of the system is to describe the occurrence of healthcare-associated infections by time and other characteristics, detect outbreaks, provide a basis for preventive measures, and to evaluate such measures. It is coordinated by the Norwegian Institute of Public Health (NIPH) in collaboration with the hospitals. The first NOIS-module encompasses SSIs following several common surgical procedures, and is described in-depth in our previous publication [3]. Data are collected during an annual 3-month surveillance period (September-November). The data are de-identified by replacing the personal identifier with a serial number before the annual submission to the NIPH. The surveillance system relies to a great extent on automatic extraction of patient data from EHRs. There are three major suppliers of electronic infection control modules (ICMs) in use in Norway. In addition some hospitals have self-developed systems, some have manual systems and some have a combined manual and electronic system.

The following NCSP surgical procedures are included in this study (in order of priority in NOIS-SSI): coronary artery bypass graft (CABG), cesarean section (CSEC), hip arthroplasty (HPRO) and cholecystectomy (CHOL). During the first few years of NOIS-SSI, exemption from submitting surveillance data was given to hospitals so that they could establish suitable ICMs. Through 2009 hospitals were required to submit data from at least one of the surgical procedures under surveillance, and from 2010 and onwards at least two procedures [3]. Mixed CABG procedures (where aorta or ventricle surgery where performed in addition to bypass) were excluded in 2008 and mixed CHOL procedures (where other procedures are performed during the same surgery) were excluded in 2007 and 2008. NOIS-SSI includes data on the following variables of interest for this study; dates of admission, discharge and surgery, NCSP codes, age, sex, and hospital identifier.

We define a hospital as a single physical unit/location. A health care trust is a legal entity, often including several hospitals. There is a trend towards hospitals reporting data on a trust level. This causes the "hospital type" to be an ambiguous categorization over time, as one trust may include several different hospital types in the latter years. We have manually categorized hospitals according to ECDC classifications [6] as follows: primary (district hospital), secondary (provincial hospital), tertiary (university hospital), and specialized (non-profit/idealistic, private, contracted hospitals that mostly perform elective surgery within certain procedure types single specialty). Hospital size was also manually categorized and is influenced by the same issues as hospital type with regard to reporting on a trust level the latter years. Regions are designated according to the official categories, South-East, West, Central and North. Type of ICM was manually coded into four categories according to whether the NOIS-SSI data for a specific year was generated from of one of the three ICM suppliers (anonymized as A, B or C to protect the identity of individual hospitals), or from a manual or inhouse system (other).

NOIS-SSI contains the patient's actual age in years on the date of surgery, but the NPR-data only provides the year of birth. To correct for this, we calculated age by generating pseudo-random birth months (1-12) and days $(1-28)$ for the NPR procedures in order to spread the patients evenly throughout the year.

In surveillance of SSIs, the denominator is the number of surgical procedures performed. One patient may undergo several procedures, such bilateral or staged hip replacement which counts as 2 procedures. The NPRdata received had one record per admission related to the surgical procedure. We converted these to one record per procedure based on the patient identifier, year of birth, sex, hospital identifier and date of surgery in order to make them comparable to SSI surveillance data. We were unable to account for bilateral hip replacements using this method, but such procedures were quite rare in Norway (0.22 \% of total hip arthroplasties in 2005-2010 [12]) and would affect our outcomes minimally. Missing procedure dates in NPR (especially 2009) were substituted by date of admission. We excluded procedures which were duplicates, had invalid surgical procedure codes or were from private clinics with inconsistent data in both registers. In addition we excluded procedures from NOIS-SSI which were outside the 3-month surveillance window, and procedures from NPR from outside 2005-2010. NOIS-SSI data were appended to NPR data for data analysis purposes. 
We evaluated the data quality of NOIS-SSI with regard to the completeness, representativeness and accuracy of the denominator data compared with NPR. We defined completeness as the total number of procedures in NOISSSI divided by the total number of procedures in NPR during the 3-month surveillance period for each procedure and year. Representativeness was assessed by comparing the distribution of data in NOIS-SSI with the distribution of data in NPR by hospital type and size, region, age and sex for each procedure and year. We defined accuracy as the agreement of data from hospitals and months which were present in both registers. We thus excluded data from hospitals or months which were not present in both registers from the comparison and divided the number of procedures in NOIS-SSI by NPR. We further compared the distributions in the two registers by the same variables as for representativeness. In addition we evaluated the accuracy based on the type of ICM used for collecting NOIS-SSI data. Frequencies were calculated for each of the surgical categories for each year, the whole period, and for each included variable. NOIS-SSI was evaluated against NPR in terms of percentages and chi-squared analysis. All data cleaning and analysis was done using Stata v.13 (Stata Statistical Software, College Station, TX). Access to deidentified data was granted to us at the discretion of the data proprietor in accordance with both registers' acts. The study has been approved by the South East Regional Committee for Medical and Health Research Ethics, and the
Norwegian Data Protection Authority has been notified. Patient consent is not required, as both NPR and NOIS are national health registers governed by separate acts.

\section{Results}

After data cleaning 162,509 procedures remained from NPR for 2005-2010, whereof 45,347 (27.9 \%) from September - November. From NOIS-SSI, 26,250 procedures were included from September-November of 2005-2010.

Table 1 shows completeness as the number of procedures submitted to NOIS-SSI divided by the total number of procedures in NPR for the 3-month surveillance period in 2005-2010. For the whole period, NOIS-SSI encompassed $57.9 \%$ of the total number of surgical procedures in NPR. The overall completeness improved from $29.2 \%$ in 2005 to $79.8 \%$ in 2010 .

Figure 1 shows the representativeness of NOIS-SSI by comparing the distribution of the procedures in NOIS-SSI with NPR by hospital size for each year. During the first years of operation NOIS-SSI differed significantly from NPR. As more hospitals submitted data during the subsequent years the distributions became more similar and thus more representative for most procedures. There was similar pattern by hospital type (data not shown), and the differences between registers cease to be significant for CABG from 2008 and for CSEC from 2009. For HPRO, only 2009 had no significant differences between the registers. For CHOL the differences are significant for all years

Table 1 Completeness: the number of procedures by type of surgical procedure and year and proportion of the procedures in NOIS versus NPR, September - November 2005-2010

\begin{tabular}{|c|c|c|c|c|c|c|c|}
\hline & 2005 & 2006 & 2007 & 2008 & 2009 & 2010 & Total \\
\hline \multicolumn{8}{|l|}{ CABG } \\
\hline NOIS & 167 & 599 & 680 & 718 & 746 & 612 & 3522 \\
\hline NPR & 1067 & 1006 & 1046 & 928 & 817 & 796 & 5660 \\
\hline Completeness & $15.7 \%$ & $59.5 \%$ & $65.0 \%$ & $77.4 \%$ & $91.3 \%$ & $76.9 \%$ & $62.2 \%$ \\
\hline \multicolumn{8}{|l|}{ CSEC } \\
\hline NOIS & 883 & 1322 & 1634 & 1948 & 2171 & 2484 & 10,442 \\
\hline NPR & 2210 & 2304 & 2443 & 2513 & 2509 & 2586 & 14,565 \\
\hline Completeness & $40.0 \%$ & $57.4 \%$ & $66.9 \%$ & $77.5 \%$ & $86.5 \%$ & $96.1 \%$ & $71.7 \%$ \\
\hline \multicolumn{8}{|l|}{ HPRO } \\
\hline NOIS & 903 & 1052 & 1338 & 1853 & 2522 & 2565 & 10,233 \\
\hline NPR & 2621 & 2628 & 2870 & 2776 & 3106 & 3141 & 17,142 \\
\hline Completeness & $34.5 \%$ & $40.0 \%$ & $46.6 \%$ & $66.8 \%$ & $81.2 \%$ & $81.7 \%$ & $59.7 \%$ \\
\hline \multicolumn{8}{|l|}{$\mathrm{CHOL}$} \\
\hline NOIS & 166 & 234 & 339 & 342 & 409 & 563 & 2053 \\
\hline NPR & 1356 & 1308 & 1394 & 1362 & 1285 & 1275 & 7980 \\
\hline Completeness & $12.2 \%$ & $17.9 \%$ & $24.3 \%$ & $25.1 \%$ & $31.8 \%$ & $44.2 \%$ & $25.7 \%$ \\
\hline TOTAL completeness & $29.2 \%$ & $44.3 \%$ & $51.5 \%$ & $64.1 \%$ & $75.8 \%$ & $79.8 \%$ & $57.9 \%$ \\
\hline
\end{tabular}

NOIS: Norwegian Surveillance System for Antibiotic Consumption and Healthcare-Associated Infections NPR: Norwegian Patient Register 


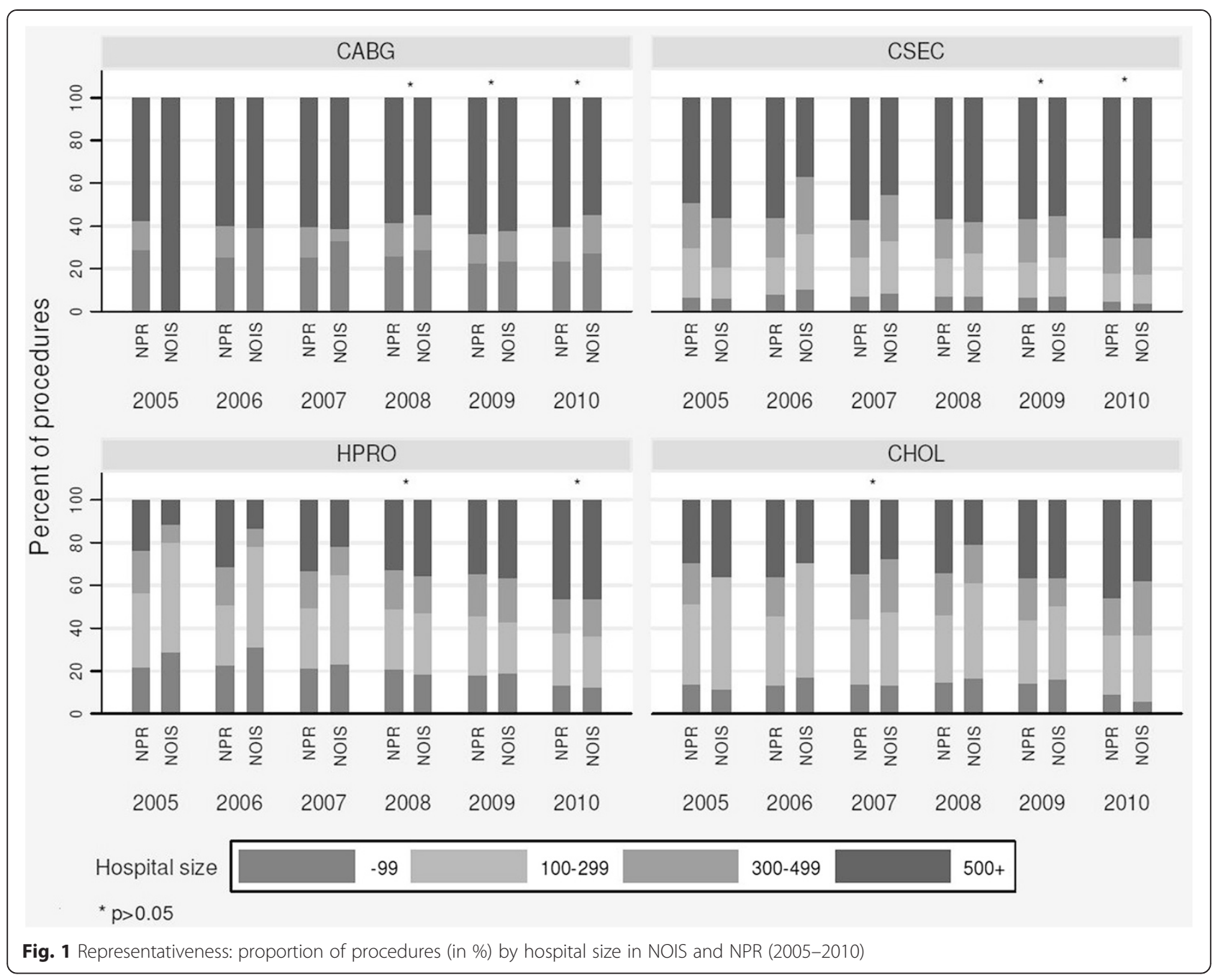

by hospital type. By region (data not shown) the differences in distribution between NOIS-SSI and NPR were greater. Only CABG in 2008 and 2009 and CSEC in 2010 had no significant differences. There were no significant differences in distribution by age and sex between NOISSSI and NPR $(p>0.05)$. The median age was about 66 for CABG, 31 for CSEC, 73 for HPRO and 49 for CHOL.

Table 2 shows the accuracy of NOIS-SSI compared with NPR by surgical procedure and year, for hospitals and reporting months which were present in both registers. Overall accuracy was $94.8 \%$, the lowest was 2008 with $90.6 \%$ and the highest was 2010 with $97.5 \%$. The procedures with the highest overall accuracy were HPRO and CSEC. There were no significant differences in distribution by region, hospital type and size, age or sex for each year and procedure $(p>0.05)$ between NOIS-SSI and NPR.

Figure 2 shows the development of ICMs from one major supplier and several manual and in-house systems in 2005, to most data from major ICM suppliers in 2010. All ICMs and other systems in the hospitals perform well, and we only find significant differences between NOIS-SSI and NPR for CSEC in $2008(p=0.001)$. System B had the highest overall accuracy $(97.5 \%)$. The three commercial systems demonstrate less variability than manual/other systems but the differences were not significant $(p>0.05)$.

\section{Discussion}

The Norwegian Surveillance System for Antibiotic Consumption and Healthcare-Associated Infections (NOIS-SSI) included $79.8 \%$ of the procedures in the administrative data during September-November 2010, up from $29.2 \%$ in 2005. NOIS-SSI was not representative with regard to hospital size and type during the earliest years, but became representative with time for some procedures. NOIS-SSI was representative with regard to age and sex for all years and procedures. The accuracy was $97.5 \%$ in 2010, an increase from $92.7 \%$ in 2005 and there were no differences in the distribution by any explanatory variables, except by type of infection control module (ICM) for CSEC in 2008. 
Table 2 Accuracy: the number of procedures by type of surgical procedure and year and proportion of the procedures in NOIS versus NPR for selected hospitals and reporting months, 2005-2010

\begin{tabular}{|c|c|c|c|c|c|c|c|}
\hline & 2005 & 2006 & 2007 & 2008 & 2009 & 2010 & Total \\
\hline \multicolumn{8}{|l|}{$\mathrm{CABG}^{1}$} \\
\hline NOIS & 167 & 402 & 519 & 580 & 503 & 520 & 2691 \\
\hline NPR & 237 & 446 & 589 & 709 & 514 & 554 & 3049 \\
\hline Accuracy & $70.5 \%$ & $90.1 \%$ & $88.1 \%$ & $81.8 \%$ & $97.9 \%$ & $93.9 \%$ & $88.3 \%$ \\
\hline \multicolumn{8}{|l|}{ CSEC } \\
\hline NOIS & 883 & 1304 & 1607 & 1826 & 2051 & 2402 & 10,073 \\
\hline NPR & 904 & 1346 & 1660 & 2014 & 2065 & 2431 & 10,420 \\
\hline Accuracy & $97.7 \%$ & $96.9 \%$ & $96.8 \%$ & $90.7 \%$ & $99.3 \%$ & $98.8 \%$ & $96.7 \%$ \\
\hline \multicolumn{8}{|l|}{ HPRO } \\
\hline NOIS & 903 & 1052 & 1338 & 1853 & 2151 & 2335 & 9632 \\
\hline NPR & 943 & 1087 & 1451 & 1959 & 2194 & 2363 & 9997 \\
\hline Accuracy & $95.8 \%$ & $96.8 \%$ & $92.2 \%$ & $94.6 \%$ & $98.0 \%$ & $98.8 \%$ & $96.3 \%$ \\
\hline \multicolumn{8}{|l|}{$\mathrm{CHOL}^{1}$} \\
\hline NOIS & 159 & 234 & 339 & 341 & 405 & 524 & 2002 \\
\hline NPR & 194 & 274 & 359 & 395 & 464 & 582 & 2268 \\
\hline Accuracy & $82.0 \%$ & $85.4 \%$ & $94.4 \%$ & $86.3 \%$ & $87.3 \%$ & $90.0 \%$ & $88.3 \%$ \\
\hline TOTAL accuracy & $92.7 \%$ & $94.9 \%$ & $93.7 \%$ & $90.6 \%$ & $97.6 \%$ & $97.5 \%$ & $94.8 \%$ \\
\hline
\end{tabular}

NOIS: Norwegian Surveillance System for Antibiotic Consumption and Healthcare-Associated Infections NPR: Norwegian Patient Register

${ }^{1}$ Mixed procedures excluded from NOIS for CABG in 2008 and for CHOL in 2007 and 2008

Comparing denominator data between two registers gives an indication of the quality of the data in both registers. It also reflects the quality of the data extraction at the individual hospital. Denominator data are important in order to reliably describe infection occurrence on a national level, in hospital benchmarking, and inter-country comparisons. Regardless of how diligent numerator (infection) case finding is, incidence proportions only make sense if the denominator data are correctly derived, giving an unbiased sample [13]. In a recent review, Goto [14] investigated the accuracy of administrative coding, but none of the included SSI-

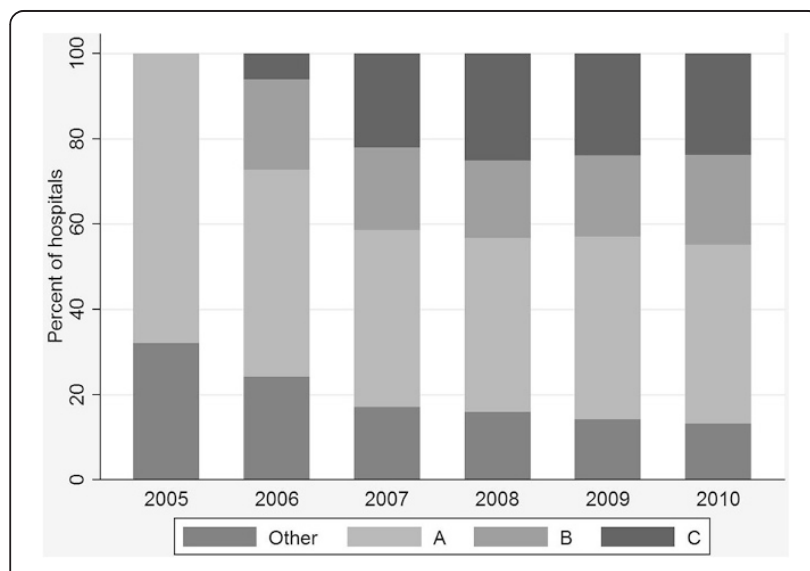

Fig. 2 Proportion of hospitals submitting data to NOIS from different electronic systems (a, b and $\mathbf{c})$ and other data sources, 2005-2010 related studies reported on the quality of denominator data. McCoubrey [15] found that $91 \%$ of eligible procedures were included in the Scottish surveillance data. Haley [16] found $98 \%$ matches between administrative- and surveillance data. Most validation studies report only on the numerator in terms of infection as outcome. A number of studies have investigated the completeness of other Norwegian health registers compared with NPR. Among these, $0.4 \%$ more CSECs were found in the Medical Birth Register of Norway [17], the Norwegian Vascular Register found a completeness of $84 \%$ for abdominal aortic aneurism repair [18], and the Norwegian Arthroplasty Register found $97 \%$ completeness of primary HPRO compared with NPR [19]. These studies are important because in addition to ascertaining the quality of the individual registers, validate the quality of NPR. Although it has improved, NOIS-SSI still only received $78.8 \%$ of the procedures performed during September-November 2010.

Because NOIS-SSI only collected data during SeptemberNovember during the study period, it was dependent on those 3 months being representative. The explanatory variables which reflect hospital participation (region, hospital type and size), show that NOIS-SSI was generally not representative for most procedures until the last years. There are several possible explanations for this.

During the first years, many hospitals were granted exemption from submitting data in order to facilitate the establishment of ICMs. The ICMs were generally purchased 
or developed for whole trusts or regions, which led to several regions submitting little or no data during the first years. Most hospitals and regions had installed ICMs by 2007, but some were not functioning optimally. This led to some hospitals and trusts being exempted also in the later years, and NOIS-SSI not being representative by region.

During 2005-2009 NOIS-SSI only required data from one procedure, the one with the highest priority. This means that hospitals were only required to submit data from the highest prioritized procedure which they performed. All hospitals which performed CABG procedures were required to submit data, but exemptions were granted to some regions and hospitals the first years. In addition, some hospitals did not submit data in later years despite it being required. If a hospital reported on CABG, it did not have to submit any other procedures. In principle this meant that none of the tertiary hospitals, which almost all performed CABG, were required to submit CSEC data causing poor representativeness by type of hospital for CSEC. This also affected representativeness by hospital size, because the tertiary hospitals are generally the largest. From 2010 a minimum of 2 procedures were required and this improved the representativeness for CSEC by hospital size and type. However, CSEC representativeness was already good in 2009, probably attributable to "enthusiastic volunteers". For HPRO, representativeness by hospital size started improving in 2008. Some of the hospitals which perform HPRO are specialized orthopedic hospitals, and these have submitted data consistently over the years. Many other hospitals have submitted HPRO data voluntarily, and this may explain why representativeness started improving before the implementation of minimum 2 procedures in 2010. For CHOL representativeness was generally poor, which is to be expected as this procedure had the lowest surveillance priority. For age and sex NOIS-SSI was representative, meaning that there were no differences between NOIS-SSI and NPR in the patient population for these variables.

In a review of four surveillance systems Haustein et al. [20] recommended mandatory reporting in order to assure that data are not biased. They found that none of the voluntary systems they investigated ever surpassed $50 \%$ participation, and that representativeness improved when reporting was made mandatory. NOIS-SSI was mandatory since inception, but a flexible implementation policy (granting exemptions) caused it not to be representative on a national level and caused participant population to change over time. The additional complication of hospitals changing from individual hospital to trust level reporting produces data which is less useful for stratification and risk purposes. This is demonstrated in Fig. 1, where a greater proportion of large hospitals are evident during the latter years. For example, 2 small primary hospitals and 1 large tertiary hospital reported individually until 2008 and from 2009 they reported as one large trust on the tertiary level.

The importance of representative surveillance data depends on how data are to be used. For evaluating risk factors and implementing preventive measures in the individual hospitals, NOIS-SSI seems to provide useful data. For hospital benchmarking and/or public reporting NOIS-SSI was not good enough, because when hospitals are not required to submit all procedures, full representativeness for such variables as hospital size and type may not be achieved.

We found the agreement between the two registers to be good, which means that when the hospitals did submit data to NOIS-SSI they appeared to be accurate. We only observed a significant difference $(p=0.001)$ between the registers by ICM for CSEC in 2008, which was mainly due to technical issues in two hospitals with the same ICMsupplier resulting in incomplete data extraction. Another reason for somewhat lower accuracy in some procedures and years was that the NOIS-SSI protocol was modified with regard to mixed procedures. The exclusion of the mixed CABG procedures in 2008 gave a dip in the accuracy of NOIS-SSI (not significant). For CHOL, exclusion of mixed procedures did not appear to influence accuracy, which is reasonable because over $90 \%$ of CHOLs were laparoscopic procedures [21] and generally not mixed (Table 2).

Automated data collection is becoming a very important tool in surveillance of HAI. It reduces the workload on hospital staff and, hopefully, human errors [22-31]. In NPR all data are collected electronically from the hospitals' EHR and in NOIS-SSI most explanatory and background variables are collected electronically from the EHR, so we could expect denominator data to be identical. However, data extraction programs may not be identical in all systems, and the syntax may differ in the way data are extracted and interpreted. In addition, NOIS-SSI data are manually checked by infection control practitioners who may manually correct the data. As demonstrated by the lower accuracy in CSEC for 2008, one cannot be certain that denominator data are correct even if they are extracted directly from hospital computer systems. Computer systems are not infallible, and it is necessary to routinely check if data are being harvested correctly. We observe some variability between the ICMs and other systems and it appears that the accuracy overall for the ICMs was more consistent than the manual/other systems, but none of the differences were significant.

The development of ICMs is complex and would have been more difficult without a flexible implementation strategy. As shown in Fig. 2, the hospitals quite quickly purchased or developed ICMs. We found the flexible 
implementation to be a double-edged sword. On one side the flexibility made good cooperation with hospitals and ICM suppliers possible and has led to quality ICMs which give good accuracy. On the other side this flexibility contributed to less representative data. Although NOIS-SSI is mandatory, the flexible implementation introduced selection bias giving poor representativeness for variables that reflect hospital participation.

NOIS-SSI improved over the first six years, but data were still not fully complete and representative in 2010 . The accuracy of NOIS-SSI was good, because the hospitals which submitted data have had consistently good denominator quality throughout the years, with a few exceptions. We also saw an indication that automated data harvesting gave slightly better denominator data quality. It is, however, difficult to assess true completeness, representativeness and accuracy without having access to linked data [32]. Being able to compare surveillance data with administrative data on a regular basis, in order to give hospitals feedback on data quality, could be a useful tool in improving quality and instilling trust in the surveillance system performance. Some have argued that administrative systems can provide more economical, standardized and unbiased outcome data than traditional surveillance systems if used correctly [33-35].

The data in this study are not linked and are compared on an aggregated level. We cannot be certain that NOIS-SSI is a subset of NPR, as both registers may contain unique records. Some variables were coded manually by the authors, and may contain unintentional errors. Birth month and date for the NPR data were generated by a pseudo-random function and does not reflect different annual birth rate patterns. For calculation of accuracy some hospitals and months were excluded from analysis, and this may give an incorrect impression of the quality of NOIS-SSI.

\section{Conclusions}

NOIS-SSI had a completeness of $79.8 \%$ of the procedures in the administrative data (NPR). The NOIS-SSI denominator data were not representative by hospital size and type during the first years of surveillance system operation, but became representative for some procedures with time. NOIS-SSI was generally not representative by region. This means that data from this period should not be used for hospital benchmarking and/or public reporting. NOIS-SSI was representative by age and sex for all procedures. For the purpose of evaluating risk factors and implementing prevention and precautionary measures in the individual hospitals, representativeness seems sufficient. Denominator data agreement between NOIS-SSI and NPR of almost $95 \%$ indicates that the accuracy of submitted data of was good. A flexible and incremental implementation strategy has encouraged development of computer-based surveillance systems in hospitals which gives good accuracy, but has adversely affected the representativeness of the data during the first years of system operation.

\section{Abbreviations}

CABG: Coronary artery bypass graft; CHOL: Cholecystectomy; CSEC: Cesarean section; ECDC: European Centre for Disease Prevention and Control; EHR: Electronic health record; HPRO: Hip arthroplasty; ICM: Infection control module; NCSP: Nordic Medico-Statistical Committee's Classification of Surgical Procedures; NIPH: Norwegian Institute of Public Health; NOIS: Norwegian Surveillance System for Antibiotic Consumption and Healthcare-Associated Infections; NPR: Norwegian Patient Register; SSI: Surgical site infection.

\section{Competing interests}

The authors declare that they have no competing interests and nothing to disclose. The project is fully financed through the budget of the Norwegian Institute of Public Health.

\section{Authors' contributions}

HLL has planned and designed the study, made substantial contributions to data collection, has analyzed and interpreted data and has drafted and revised the manuscript. HME has been involved in the planning and design of the study, has been involved in data collection and participated in the analysis and interpretation of data. PA has been involved in the planning and design of the research study. FES has made a substantial contribution to the planning and design of the research study and has made a substantial contribution to data acquisition. All authors have substantially contributed to the draft and revising of the manuscript critically and have approved the final version.

\section{Acknowledgements}

The authors thank the infection control practitioners at Norwegian hospitals for being the driving force and coordinating link in NOIS. We also thank the Norwegian Patient Register for supplying data.

\section{Author details}

${ }^{1}$ Norwegian Institute of Public Health, Department of Infectious Disease Epidemiology, Oslo, Norway. ${ }^{2}$ Epidemi, Lasarettet, Kristiansand, Norway. ${ }^{3}$ Faculty of Health Sciences, Department of Community Medicine, Research Group Epidemiology of Chronic Diseases, UiT The Arctic University of Norway, Tromsø, Norway.

Received: 14 April 2015 Accepted: 24 November 2015

Published online: 30 November 2015

\section{References}

1. ECDC. Surveillance of surgical site infections in Europe 2010-2011. In: ECDC Survellance report. Stockholm: European Centre for Disease Prevention and Control; 2013.

2. HOD. Norwegian Surveillance System for Antibiotic Consumption and Healthcare-Associated Infections (NOIS) Act. Oslo: Lovdata, Ministry of Health and Care Services; 2005.

3. Lower HL, Eriksen HM, Aavitsland P, Skjeldestad FE. Methodology of the Norwegian Surveillance System for Healthcare-Associated Infections: the value of a mandatory system, automated data collection, and active postdischarge surveillance. Am J Infect Control. 2013;41(7):591-6.

4. Lower HL, Dale H, Eriksen HM, Aavitsland P, Skjeldestad FE. Surgical site infections after hip arthroplasty in Norway, 2005-2011: influence of duration and intensity of postdischarge surveillance. Am J Infect Control. 2015;43(4):323-8.

5. HELICS. Hospitals in Europe Link for Infection Control through Surveillance. In: Surveillance of Surgical Site Infections Protocol v. 9.1. 2004.

6. ECDC. Surveillance of surgical site infections in European hospitals- HAISSI protocol. V 1.02. Stockholm: European Centre for Disease Prevention and Control; 2012.

7. Horan TC, Gaynes RP, Martone WJ, Jarvis WR, Emori TG. CDC definitions of nosocomial surgical site infections, 1992: a modification of CDC definitions of surgical wound infections. Infect Control Hosp Epidemiol. 1992;13(10):606-8.

8. German RR, Lee LM, Horan JM, Milstein RL, Pertowski CA, Waller MN, Guidelines Working Group Centers for Disease C, Prevention. Updated 
guidelines for evaluating public health surveillance systems: recommendations from the Guidelines Working Group. MMWR Recomm Rep. 2001;50(RR-13):1-35. quiz CE31-37.

9. HOD. The Norwegian Patient Register Act. Oslo: Lovdata, Ministry of Health and Care Services; 2012.

10. Bakken IJ, Nyland K, Halsteinli V, Kvam UH, Skjeldestad FE. Norsk pasientregister: Administrativ database med mange forskningsmuligheter. Norsk Epidemiologi. 2004;14(1):65-9.

11. NOMESCO. NOMESCO Classification of Surgical Procedures (NCSP), version 1.16. Copenhagen: Nordic Medico-Statistical Committee (NOMESCO); 2009

12. NAR: Report June 2015. In.Bergen: Norwegian Arthroplasty Register; 2015

13. Woeltje KF, Lin MY, Klompas M, Wright MO, Zuccotti G, Trick WE. Data requirements for electronic surveillance of healthcare-associated infections. Infect Control Hosp Epidemiol. 2014;35(9):1083-91.

14. Goto M, Ohl ME, Schweizer ML, Perencevich EN. Accuracy of administrative code data for the surveillance of healthcare-associated infections: a systematic review and meta-analysis. Clin Infect Dis. 2014;58(5):688-96.

15. McCoubrey J, Reilly J, Mullings A, Pollock KG, Johnston F. Validation of surgical site infection surveillance data in Scotland. J Hosp Infect. 2005;61(3): 194-200.

16. Haley VB, Van Antwerpen C, Tserenpuntsag B, Gase KA, Hazamy P, Doughty $D$, et al. Use of administrative data in efficient auditing of hospital-acquired surgical site infections, New York State 2009-2010. Infect Control Hosp Epidemiol. 2012;33(6):565-71.

17. Backe B, Heggestad T, Lie T. The epidemic of Caesarean section: has it reached Norway? Tidsskr Nor Laegeforen. 2003;123(11):1522-4.

18. Haug ES, Romundstad P, Saether OD, Jorgenvag R, Myhre HO. Quality of data reported on abdominal aortic aneurysm repair-a comparison between a national vascular and a national administrative registry. Eur J Vasc Endovasc Surg. 2005;29(6):571-8.

19. Espehaug B, Furnes O, Havelin LI, Engesaeter LB, Vollset SE, Kindseth O. Registration completeness in the Norwegian Arthroplasty Register. Acta Orthop. 2006;77(1):49-56

20. Haustein T, Gastmeier P, Holmes A, Lucet J-C, Shannon RP, Pittet D, et al Use of benchmarking and public reporting for infection control in four high-income countries. Lancet Infect Dis. 2011;11(6):471-81.

21. Bakken IJ, Skjeldestad FE, Mjaland O, Johnson E. Cholecystectomy in Norway 1990-2002. Tidsskr Nor Laegeforen. 2004;124(18):2376-8.

22. Daneman N, Ma X, Eng-Chong M, Callery S, Guttmann A. Validation of administrative population-based data sets for the detection of cesarean delivery surgical site infection. Infect Control Hosp Epidemiol. 2011;32(12): $1213-5$.

23. Leal J, Laupland KB. Validity of electronic surveillance systems: a systematic review. J Hosp Infect. 2008;69(3):220-9.

24. Leth RA, Norgaard M, Uldbjerg N, Thomsen RW, Moller JK. Surveillance of selected post-caesarean infections based on electronic registries: validation study including post-discharge infections. J Hosp Infect. 2010; 75(3):200-4

25. Greene LR, Cain TA, Khoury R, Krystofiak SP, Patrick M, Streed S. APIC position paper: The importance of surveillance technologies in the prevention of health care-associated infections. Am J Infect Control. 2009;37(6):510-3.

26. Bolon MK, Hooper D, Stevenson KB, Greenbaum M, Olsen MA, Herwaldt L, et al. Improved surveillance for surgical site infections after orthopedic implantation procedures: extending applications for automated data. Clin Infect Dis. 2009:48(9):1223-9.

27. Gerbier-Colomban S, Bourjault M, Cetre JC, Baulieux J, Metzger MH. Evaluation study of different strategies for detecting surgical site infections using the hospital information system at Lyon University Hospital. France Ann Surg. 2012;255(5):896-900.

28. Trick WE. Decision making during healthcare-associated infection surveillance: a rationale for automation. Clin Infect Dis. 2013;57(3):434-40

29. van Mourik MS, Troelstra A, van Solinge WW, Moons KG, Bonten MJ. Automated surveillance for healthcare-associated infections: opportunities for improvement. Clin Infect Dis. 2013;57(1):85-93.

30. Weiskopf NG, Weng C. Methods and dimensions of electronic health record data quality assessment: enabling reuse for clinical research. J Am Med Inform Assoc. 2013;20(1):144-51.

31. Yokoe DS, Khan Y, Olsen MA, Hooper DC, Greenbaum M, Vostok J, et al. Enhanced surgical site infection surveillance following hysterectomy, vascular, and colorectal surgery. Infect Control Hosp Epidemiol. 2012;33(8):768-73.
32. Bakken IJ, Suren P, Haberg SE, Cappelen I, Stoltenberg C. The Norwegian patient register-an important source for research. Tidsskr Nor Laegeforen. 2014;134(1):12-3.

33. Calderwood MS, Ma A, Khan YM, Olsen MA, Bratzler DW, Yokoe DS, et al. Use of Medicare diagnosis and procedure codes to improve detection of surgical site infections following hip arthroplasty, knee arthroplasty, and vascular surgery. Infect Control Hosp Epidemiol. 2012;33(1):40-9.

34. Klompas M, Yokoe DS. Automated surveillance of health care-associated infections. Clin Infect Dis. 2009;48(9):1268-75.

35. Wright MO. Automated surveillance and infection control: Toward a better tomorrow. Am J Infect Control. 2008;36(3):S1-6.

\section{Submit your next manuscript to BioMed Central and we will help you at every step:}

- We accept pre-submission inquiries

- Our selector tool helps you to find the most relevant journal

- We provide round the clock customer support

- Convenient online submission

- Thorough peer review

- Inclusion in PubMed and all major indexing services

- Maximum visibility for your research

Submit your manuscript at www.biomedcentral.com/submit 